\title{
A Review of Speech Rhythm Pattern in Second Language Acquisition
}

\author{
Yingxue Zhang \\ Xinjiang Normal University, Urumqi 830017, Xinjiang, China
}

Funding: Funded by Postgraduate Research \& Practice Innovation Program of Xinjiang Normal University; Project Name: An Acoustic Study of Standard Chinese Speech Rhythm by Preparatory University Students--A Case Study of Xinjiang Normal University; Project number: XSY202001014.

\begin{abstract}
As an important prosodic feature in the processing of natural languages, speech rhythm refers to the temporal organization of language. The present study reviewed the literature related to speech rhythm pattern of second language. It is indicated most of the previous studies only focused on the ESL learners' speech rhythm patterns. However, there are a few studies on the rhythm patterns of Mandarin Chinese learners. Thus, it is necessary to investigate the rhythmic patterns of different second language learners because rhythmic cues are important to evaluate the degree of accentedness in speech.
\end{abstract}

Keywords: Second language; Rhythm; Rhythmic metrics

Publication date: April, 2021; Publication online: 30 April, 2021

*Corresponding author: Yingxue Zhang, 1006424356@qq.com

\section{Introduction}

The word "rhythm" can be defined from different perspectives. From the perspective of phonetics, speech rhythm has been defined as a periodic recurrence of events and as a structural property of languages by which they can be allocated to different rhythm classes or be located along a rhythmic continuum ${ }^{[1]}$. Traditionally, languages were labeled as either syllable-timed, stress-timed, or mora-timed in terms of speech rhythm ${ }^{[2]}$. Therefore, relevant metrics have been proposed to quantify these speech rhythmic patterns and the differences between stress-timed languages and syllabletimed languages have been listed in the studies of researchers. In recent years, these quantitative indicators are gradually used in the field of second language acquisition to explore the characteristics of learners' language rhythm. Since the main function of rhythm is to form words and sentences according to the needs of semantic expression or understanding when speaking and listening, and the needs of semantic expression and understanding can be expressed in a rhythmic structural mode. The purpose of this study is to review the related to speech rhythm of second language. The provide a reference for the study of second language rhythm characteristics from three aspects: the definition of rhythm, the quantitative rhythmic indicators and the related researches of second language rhythm characteristics.

\section{Previous Studies on L2 Acquisition of Speech}

\section{Rhythm Abroad}

\subsection{English as L2 Acquisition of Speech Rhythm}

Wenk found that the rhythmic pattern of English produced by the French native speakers always influenced by their mother tongue, and transfer it to English learning. In his point of view, as to the foreign language learners, only by overcoming the influence of mother tongue can they improve their English proficiency ${ }^{[3]}$.

Low et al have taken researches on the rhythm of Singapore English learners which is influenced by Chinese both in continuous speech and in conversational speech. By comparing data based on the vowel quality, they showed that Singapore English is indeed more syllable-timed. The results revealed that successive vowel duration is earlier equal in Singapore English than in British English ${ }^{[4]}$.

In Mimatsu's study, he found that some of the subjects were able to produce the rhythm patterns similar to English 
that was different from Japanese. Typically, they are more or less unable to reduce vowels without stress. As a result, each syllable they produced weighs as much as in Japanese ${ }^{[5]}$.

Mok and Dellwo compared the rhythmic patterns of English spoken by Mandarin and Cantonese native speakers ${ }^{[6]}$.

Grenon and White compared the rhythm between native Canadian English and Japanese English, and found that rhythm production in English by Japanese speakers were comparable to native English speakers on all metrics $(\% \mathrm{~V}$, Varco V and rPVI_C) Despite these results, they found that Japanese speakers were not aware of the stressed-unstressed contrast that the Varco $\mathrm{V}$ scores are to reflect like native English speakers ${ }^{[7]}$.

Sarmah, Gogio, and Wiltshire used \%V and nPVI_V metrics to evaluate the rhythm patterns in English by native Thai learners in the United States. They found that learners who studied in the United States for less than 6 months showed higher nPVI_V and lower \%V values compared to learners who had studied more than 18 months in the United States. What's more, the group that had spent more time studying in the United States showed more distant values from native English ones ${ }^{[8]}$.

Peggy P. K. M. studied the speech rhythm by CantoneseEnglish bilingual children and their age-matched monolingual peers. In this study, six Cantonese-English bilingual children aged about 3 were compared with six monolingual children in each language by using mostly used acoustic rhythmic metrics on consonantal, vocalic, and syllabic intervals. His study also included qualitative data on syllable structure complexity and vowel quality. In this study, on syllable duration monolingual children display different rhythmic patterns, while between the two languages of the bilingual children the differences are less distinct. Monolingual English has more durational variability than bilingual English. There is a distinct phonological developmental trajectory between bilingual and monolingual children, which is influenced by language dominance. This suggests that the two phonologies interact at the prosodic level ${ }^{[9]}$.

Ordin et al chose German (native language) and English (target language) learners as subjects and when they exhibit similar rhythmic properties, the durational variability in the speech of German learners also increases with the growth of proficiency ${ }^{[10]}$. The common phonological parameters between English and German are known to influence rhythmic measurement. In terms of phonetic timing patterns captured by metric scores, German and English are classified as stress-timed ${ }^{[11]}$ and exhibit typical phonological characteristics of stress-timed languages ${ }^{[12]}$. Therefore, there is no need for German learners of English to acquire such phonological characteristics like the opposition between long and short vowels, production of complex syllables and complex consonantal clusters, etc.

Ordin and Polyanskaya found that in both children speaking and in adults learning other languages, the speech rhythm of language develops from more syllable-timed toward more stress-timed patterns and then develop languagespecific rhythmic patterns of the target language using spontaneous speech and uncontrolled as speech material. The study has shown that the rhythmic patterns of native English learners with syllable-timed native languages become increasingly more stress-timed as a function of the length of residence ${ }^{[13]}$.

$\mathrm{Li}$ and Post studied the development of speech rhythm in typologically different languages from native language at different proficiency levels. He empirically investigated the rhythmic patterns produced by German and Mandarin learners of English at either B1 or C1 proficiency levels. They found that compared to advanced learners, both German and Chinese learners at the intermediate level produced a lower degree of durational variability in speech $^{[14]}$.

\subsection{Other Languages as L2 Acquisition of Speech Rhythm}

Guilbault compared the rhythmic properties in spontaneous speech between experienced and inexperienced French learners with Canadian English as their native language. He used the variability index (VI) metric to calculate the durational differences in average between a stretch of successive syllables. He found that inexperienced learners tended to have lower VI than that of the experienced ones, but the difference between the two groups was not much significant. Both the target language proficiency and the time spent in the target language environment seemed to have no significant effect on the target language rhythm of learners' $\operatorname{speech}^{[15]}$.

Stockmal et al. conducted a study concerning rhythm in the speech of advanced Russian learners of learning Latvian and did not find any significant increase in vocalic variability between advanced and low-level Russian learners, despite the fact that Latvian is significantly less stress-timed than Russian. This shows that in the early stages of language learning, low vocalic variability in the target language corresponds to low 
vocalic variability even when the native language of the learner is stress-timed and therefore characterized by a higher degree of durational variability. If a target language is also characterized by a low degree of durational variability, then the rhythm metrics of vocalic intervals do not increase during the language learning process ${ }^{[16]}$.

\section{Previous Studies on L2 Acquisition of Speech}

\section{Rhythm at Home}

\subsection{English as L2 Acquisition of Speech Rhythm}

Zhu argued that rhythmic patterning was an important prosody feature. In his study, he investigated the similarities and differences of English and Mandarin Chinese rhythmic patterns, and put forward some modes of English rhythm patterns suitable for English teaching ${ }^{[17]}$.

According to the results of Lin and Wang's research, it shows that the four measures, i.e., $\Delta \mathrm{C}, \% \mathrm{~V}, \mathrm{rPVI} C \mathrm{C}, \mathrm{nPVI} \mathrm{V}$, as a whole cannot explain the Mandarin Chinese EFL learners' rhythm; instead, each measure should be analyzed individually in order to achieve a complete understanding and a full characterization of the ESL rhythm ${ }^{[18]}$.

$\mathrm{Yu}$ investigated the rhythm patterns in the speech of Chinese EFL learners, especially those with Hangzhou dialect. By focusing on the prominence and timing pattern, it compared the rhythmic differences in English reading speech between English natives and Chinese EFL learners. With the help of Chinese dialectology and phonetic methodology, it also analyzed and interpreted the underlying reasons for the problems observed from the rhythm patterns of Chinese EFL learners. It aims to facilitate English phonetic teaching, establish an automatic English pronunciation evaluation system and provide acoustic parameters for speech engineering ${ }^{[19]}$.

\subsection{Chinese as L2 Acquisition of Speech Rhythm}

Zhou investigated Chinese rhythm by American students. They found that American students of intermediate Chinese level did not acquire the rhythmic patterns of Chinese, which led to their foreign accent ${ }^{[20]}$.

Gu conducted a study comparing the rhythm patterns of native Mandarin learners, native Cantonese learners, and native English learners. The study starts with isolated words with neutral-toned syllables, focuses on continuous speech flow, and objectively analyzes rhythm patterns by measuring 11 duration metrics. According to the study of continuous speech flow, it is found that Mandarin spoken by Cantonese and English learners shows a rhythm pattern very close to that of native Mandarin based on the duration index that is irrelevant to or normalized to the speed of speech, while Mandarin moves towards the stress rhythm pattern based on the duration index that is not normalized to the speed of speech, which is irrelevant to the rhythm pattern of a native speaker $^{[21]}$.

\section{Conclusion}

This paper makes a literature review of second language rhythm acquisition at home and abroad. On the whole, there are many researches on the characteristics of second language rhythm abroad. In addition, no matter abroad or at home, most of researches focus on the second language learners of English. The research corpus includes read speech and spontaneous speech, which are in the form of words, sentences, texts, etc. by measuring a series of different rhythm metrics. In the future, the study of second language rhythm patterns can involve more second language learners of other language types. What's more, the reliability of rhythm metrics could be confirmed in more and more studies of second language learners.

\section{References}

[1] Lloyd, J. A. Speech Signals in Telephony[M]. London: Sir I. Pitman and Sons, 1940.

[2] Abercrombie, D. Elements of General Phonetics[M]. Edinburgh: Edinburgh University Press, 1967.

[3] Wenk, B. Crosslinguistic influencein second language phonology: Speech rhythms, 1986: 120-133.

[4] Low, E. L. Prosodic prominence in Singapore English (Unpublished doctoral dissertation). University of Cambridge, 1998.

[5] Mimatsu, K. An essay on incorrect Eglish pronunciation of text reading by Japanese 135 college students, 2000.

[6] Mok, P. and Dellwo, V. Comparing native and nonnative speech rhythm using acoustic rhythmic measures: Cantonese, Beijing Mandarin and English[C]. the 4th Speech Prosody, 2008: 423-426

[7] Grenon, I.and White, L., How stable are acoustic metrics of contrastive speech rhythm? [J]. J. Acoust. Soc. Am, 2010.127, 1559-1569.

[8] Sarmah, P., Gogio, D. V., and Wiltshire, C. Thai English: Rhythm and vowels[J]. English World-Wide, 2009, 30:196-217

[9] Peggy, P. K. M. The acquisition of speech rhythm by 
three-year-old bilingual and monolingual children: Cognition, 2011, 14(04):458-472.

[10] Ordin, M., Polyanskaya, L. and Ulbrich, C. Acquisition of timing patterns in second language, in Interspeech 2011, Florence, ISCA, 2011:1129-1132.

[11] Grabe, E. and Low, E. L. Durational variability in speech and the rhythm class hypothesis[D]. Berlin, New York: Mouton de Gruyter, 2002:515-546.

[12] Dauer, R. M. Phonetic and phonological components of language rhythm[J]. In Proceedings of the 11th ICPhS, 1987:447-450.

[13] Ordin, M. and Polyanskaya, L. Development of timing patterns in first and second languages[M]. System, 2014, 42: 244-257.

[14] Li, A. and Post, B. L2 acquisition of prosodic properties of speech rhythm[M]. Stud. Second Lang. Acquis, 2014: 36(2), 223-255.

[15] Guilbault, C. P. G. The acquisition of French rhythm by English second language learners (Unpublished doctoral dissertation). University of Alberta, Edmonton, 2002.

[16] Stockmal, V., Markus, D., and Bond, D. Measures of
Cantonese and English[J]. Bilingualism Language and native and non-native rhythm in a quantity language[J]. Language and Speech, 2005, 48: 55-63.

[17] Zhu Lin.A Comparison of English and Chinese Rhythmic Patterns -- and International Forum on Frontier Issues in Phonetics. The 7th Chinese Phonetics Academic Conference \& International Forum on Frontier Issues in Phonetics,2006:1-9.

[18] Lin Hua, Wang Qian.Mandarin Rhythm: An Acoustic Study [J]. Journal of Chinese Linguistics and Computing, 2007,17(3):127-140.

[19] Yu Jue.A study on the rhythm pattern of Chinese students' English reading [D]. Zhejiang University,2013.

[20] Zhou Shiping.Chinese Language Teaching and Research, 2008.000(002):38-44,63. (in Chinese)

[21] Gu Wentao, Liu Xueyuan, Hirose Keiyoshi.An Experimental Study on the Rhythmic Pattern of Mandarin as a Second Language [J]. Journal of College of Liberal Arts of Nanjing Normal University, 2014(3):169-175. 